\title{
Therapeutic Perspectives on Chia Seed and Its Oil: A Review
}

\author{
Authors \\ John Parker ${ }^{1}$, Amanda N. Schellenberger ${ }^{1}$, Amy L. Roe ${ }^{2}$, Hellen Oketch-Rabah ${ }^{3}$, Angela I. Calderón ${ }^{1}$ \\ Affiliations \\ 1 Department of Drug Discovery and Development, Harri- \\ son School of Pharmacy, Auburn University, Auburn, AL \\ 2 The Procter \& Gamble Company, Cincinnati, $\mathrm{OH}$ \\ 3 United States Pharmacopeial Convention, Rockville, MD \\ Key words \\ chia seed, chia seed oil, Salvia hispanica, biological activity, \\ $\alpha$-linolenic acid, Lamiaceae \\ received September 18, 2017 \\ revised February 23, 2018 \\ accepted March 1, 2018 \\ Bibliography \\ DOI https://doi.org/10.1055/a-0586-4711 \\ Published online March 13, 2018 | Planta Med 2018; 84: 606- \\ 612 (c) Georg Thieme Verlag KG Stuttgart · New York I \\ ISSN 0032-0943 \\ Correspondence \\ Dr. Angela I. Calderón \\ Department of Drug Discovery and Development, Harrison \\ School of Pharmacy, Auburn University \\ 4306 B Walker Building, 36849 Auburn, Alabama, USA \\ Phone: + 13348448333 , Fax: + 13348448331 \\ aic0001@auburn.edu

\begin{abstract}
The attraction of novel foods proceeds alongside epidemic cardiovascular disease, diabetes, obesity, and related risk factors. Dieticians have identified chia (Salvia hispanica) as a product with a catalog of potential health benefits relating to these detriments. Chia is currently consumed not only as seeds, but also as oil, which brings about similar effects. Chia seeds and chia seed oil are used mainly as a food commodity and the oil is also used popularly as a dietary ingredient used in various dietary supplements available in the U.S. market. Chia seed is rich in $\alpha$-linolenic acid, the biological precursor to eicosapentaenoic acid, a polyunsaturated fatty acid, and docosahexaenoic acid. Because the body cannot synthesize $\alpha$-linolenic acid, chia has a newfound and instrumental role in diet. However, the inconclusive nature of the scientific community's understanding of its safety warrants further research and appropriate testing. The focus of this work is to summarize dietary health benefits of $S$. hispanica seed and oil to acknowledge concerns of adverse events from its ingestion, to assess current research in the field, and to highlight the importance of quality compendial standards to support safe use. To achieve this end, a large-scale literature search was partaken on the two well-known databases, PubMed and SciFinder. Hundreds of articles detailing such benefits as decreased blood glucose, decreased waist circumference and weight in overweight adults, and improvements in pruritic skin and endurance in distance runners have been recorded. These benefits must be considered within the appropriate circumstances.
\end{abstract}

\section{Introduction}

Chia (Salvia hispanica L.) is a summer annual herbaceous flowering plant belonging to the Lamiaceae family observed to flower with purple and/or white petals found in southern Mexico, where its seeds have been consumed by local peoples since antiquity [1]. It is native to central and southern Mexico and Guatemala, where it has historically been regarded as an essential food ingredient and is still used as a food and in beverages [2,3]. The USP defines chia seed oil as the oil extracted from the seeds by cold pressing and excludes the use of solvents or external heat in the extraction process. For purposes of preserving the oil, tocopherols may be added as antioxidants [2]. Because of the increasing use of chia seed oil in dietary supplements, the USP recently developed a public quality standard for chia seed oil to provide dietary supple- ment manufacturers with a measure to ensure that they can deliver quality products to the public, if they choose to conform to the USP standard [2]. The development of USP dietary supplement quality standards is preceded by an admission evaluation that considers various sets of information about an article proposed for monograph development, including the history of use, safety related data, animal pharmacological data, regulatory data, among others, to determine whether the article is associated with signals of safety concern. The article is admitted to the monograph development process if it is not associated with signals of safety concern or if it is associated with only mild adverse reactions that can be mitigated by a label caution statement. Chia seed passed the USP admission evaluation, and the information discussed in this review was considered during the admission of chia seed oil for USP monograph development [2]. It is important 


$\begin{array}{ll}\text { ABBREVIATIONS } \\ \text { ALA } & \alpha \text {-linolenic acid } \\ \text { ALT } & \text { alanine aminotransferase } \\ \text { AST } & \text { aspartate aminotransferase } \\ \text { DHA } & \text { docosahexaenoic acid } \\ \text { DHFR } & \text { dihydrofolate reductase } \\ \text { EPA } & \text { eicosapentaenoic acid } \\ \text { GSH } & \text { glutathione } \\ \text { GSSH } & \text { glutathione reductase } \\ \text { HSP } & \text { heat shock protein } \\ \text { ISAC } & \text { International Society for Analytical Cytology } \\ \text { MCHC } & \text { mean corpuscular hemoglobin concentration } \\ \text { MCV } & \text { mean corpuscular volume } \\ \text { MUFA } & \text { monounsaturated fatty acid } \\ \text { NEFA } & \text { non-esterified fatty acid } \\ \text { PUFA } & \text { polyunsaturated fatty acid } \\ \text { SCD } & \text { stearoyl-CoA desaturase } \\ \text { USP } & \text { United States Pharmacopeia }\end{array}$

to note that the monograph is official since December 2017. The European Union recognized S. hispanica as a novel food since 2009, and in the United States chia seed is generally recognized as safe, although no such distinction exists for chia see oil [4]. In Canada, chia seeds and oil are listed as a "natural health product ingredient" [4]. In the Eastern hemisphere, Australia sees wide consumption by its citizens; though it is not officially listed as a medicinal ingredient. No data were found for China [5].

\section{Literature search technique}

The review is a result of relevant literature searches conducted on PubMed and SciFinder on S. hispanica ( $\bullet$ Fig. 1). In SciFinder and PubMed, "chia seed" and "chia seed oil" were searched and sorted by natural order. To retrieve in vitro studies, the search "chia seed in vitro" was employed to reduce extraneous results. The sole criteria for exclusion and inclusion of references had to do with therapeutic relevancy; neither year published nor language was used as a parameter for inclusion or exclusion as evidenced by the presence of articles originally written in Spanish and other languages (the reports ranged from 2000 to 2017). No extra method was used to identify relevant animal studies.

\section{PubMed technique}

"Chia seed" was searched to avoid authors bearing the last name "Chia" ( $n=154)$ along with "Salvia hispanica L." ( $n=56)$ and then less specifically "Salvia hispanica" ( $\mathrm{n}=76)$.

\section{SciFinder technique}

The following procedure was used in SciFinder. "Chia seed" $(n=373)$ was searched just as in PubMed, but relevant sources were filtered by using the Analyze Tab, CA Section Title, Show More, and Sort by Natural Order (which uses Natural Language Processing), at which point the reports were handpicked for relevance to in vitro, in vivo, or human studies.
Reports that focused on physical characteristics of chia seed or chia seed oil in both search engines were not included in the manuscript. Of the reports, only three out of the 54 were review papers while the remaining 51 were original research papers. Of the 51 original research papers, 21 were clinical trials, 17 were in vivo studies on animals, and three were in vitro studies. All languages were included in the search criteria. As the use of Salvia is a relatively new development, most hits come from the most recent years; however, reports were neither included nor excluded based on year or content. A systematic review by Ullah et al. [6] brings to light much organized information on the state of research at its time of publication in 2016. However, it has been demonstrated by the researchers of the current project that the present manuscript places a particular focus on therapeutic measures especially within humans, whereas Ullah et al. [6] took a particular food science and technological view of the status of research in this topic. The present manuscript highlights positive, negative, and neutral information on the current state of scientific literature involving chia on human health, with supporting findings in animal studies to substantiate the current knowledge.

\section{Phytochemical Description of Chia Seed and Oil}

The major constituents of chia seed oil include essential fatty acids. Chia seeds oil is rich in PUFAs, specifically omega-3 linolenic acid (54-67\%) and omega-6 linoleic acid (12-21\%) [7]. Chia seed exhibits the following chemical characteristics, and percentages reflect mass percentage per $100 \mathrm{~g}$ of chia seed. Moisture and ash compose $6.38 \%$ and $4.82 \%$ of the total mass, respectively, and protein constitutes $18.95 \%$ of the seed. The total carbohydrate component was $42.12 \mathrm{~g} / 100 \mathrm{~g}$, and the total dietary fiber is valued $34.4 \mathrm{~g} / 100 \mathrm{~g}$ [8]. The total energy value was determined to be $369.93 \mathrm{kcal}$ per $100 \mathrm{~g}$ chia seed. However, the lipid category provides the most essential benefits of chia consumption. Lipids make up $31.34 \%$ of the total mass of the seed, with the polyunsaturated variety, the monounsaturated variety, and saturated fats making up $26.74 \%, 1.79 \%$, and $2.81 \%$ of the total seed mass, respectively. Out of the total lipid component, $19.56 \%$ comprised 18:3 (n-3) polyunsaturated fats, which includes ALA, the biological precursor to EPA and DHA. Imran et al. [9] tested a sample of chia seeds that contained $6.16 \%$ moisture, $34.84 \%$ oil, $18.21 \%$ protein, $4.16 \%$ ash, $23.12 \%$ fiber, and $14.18 \%$ nitrogen contents. It has been demonstrated that a high concentration of $\mathrm{n}-3$ is associated with a lower risk of coronary artery disease, hypertension, type 2 diabetes, rheumatoid arthritis, autoimmune disorders, and cancer $[10,11]$.

\section{Health Benefits Attributed to Chia Seeds and Oil}

\section{Antiproliferative and apoptotic effects}

Seed oils rich in n-3 and n-6 PUFAs may inhibit the growth of breast tumor cells in animals. In Wistar rats administered $25 \mathrm{~g}$ of 
chia seed or powder mixed with $0.25 \mathrm{~L}$ of water twice daily, tumor weight was significantly decreased in the chia oil group compared to the control but not in comparison to the safflower oil group. Thus, chia oil diet reduced tumor weight, encouraged apoptosis, and discouraged mitosis. The membranes from the Carthamus tinctorius L. (Compositae), commonly known as safflower oil, diet contained a greater percentage of ALA with respect to control and chia oil. Chia oil diet increased EPA the most. Espada et al. [12] proposed that EPA might have a direct apoptotic effect by encouraging caspase activity, increasing the rate of programmed cell death. Given the previous claim of EPA's role in encouraging caspase activity, chia or similarly constituted seeds may have unpredicted antitumor potential; however, the limitation that chia did not make significant improvements compared to the safflower oil group must be emphasized. Additionally, a 2017 cytotoxicity study by Rosas-Ramirez et al. [13] examined the effect of chia oligosaccharide mucilages in modifying the resistance and decreasing proliferation of cancerous cells, especially HeLa, MCF7, and HepG2 cells. The author of this study points out that the assurance that these mucilages do not attack noncancerous cells has yet to be achieved.

\section{Immunostimulant effects}

Ayerza and Coates assessed immune stimulant activity of chia and concluded that the product has strategic advantages over other omega-3 PUFA products in relieving conditions such as diarrhea, allergies, fishy flavor, weight loss, and digestive problems [14]. Chia was found to have a comparable effect as fish oil in such parameters as food intake, body weight, thymus weight, thymocyte number, and immunoglobin E levels [14]. This study was unique in its focus on the immune system, and a glaring shortcoming of the current knowledge of chia immunostimulant effects is that none of the findings have been replicated.

\section{Effects on the cardiovascular system}

In vitro antioxidant and antihypertensive activity

Rahman et al. [15] conducted a study that highlights S. hispanica's antioxidative potential. Chia oil olein fractions at percentages $5 \%$, $10 \%, 15 \%$, and $20 \%$ replaced the control ( $100 \%$ milk fat) in the experimental group and improved the 2,2-diphenyl-1-picrylhydrazyl free radical scavenging activity of control at percentages of $5.61 \%, 17.43 \%, 36.84 \%, 51.17 \%$, and $74.91 \%$, respectively (control to test article groups 1-4). Hydrolysis of chia produces bioactive peptides with low molecular weight. These molecules demonstrated angiotensin-converting enzyme inhibitory and antioxidant activity [16]. Another study that tested the low molecular weight fraction obtained through ultrafiltration also showed bioactivity in angiotensin-converting enzyme inhibitory potential [17]. A study conducted by Salazar-Vega et al. [18] showed that protein hydrolysates from chia seeds had an inhibitory effect on angiotensin-converting enzyme that was several times more powerful than Phaseolus lunatus L. (Fabaceae) and Phaseolus vulgaris L. (Fabaceae). This suggests that chia acts as an electron donor and free radical scavenger providing antioxidant protection that may translate to antihypertensive activity [18]. Antioxidant and potential antihypertensive effects were also implied in a study by Orono-Tamayo et al. [19], in which a gastrointestinal simulation

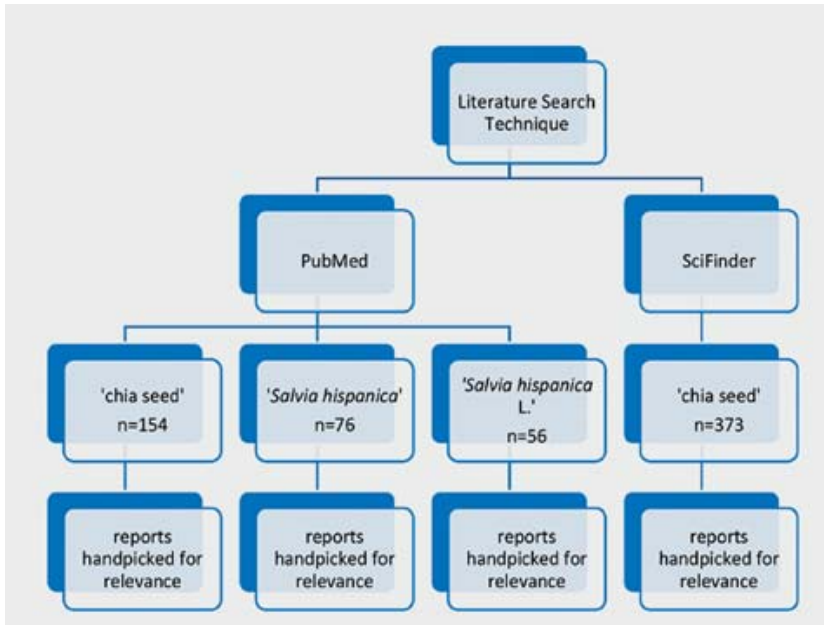

- Fig. 1 Literature search technique.

with chia resulted in a high antiradical activity against 2,2'-azinobis(3-ethylbenzothiazoline6-sulfonic acid), 2,2-diphenyl-1-picrylhydrazyl, and angiotensin-converting enzyme activity. Prolamin and globulin fraction peptides from chia were most successful in chelating the ferrous ion, also tested in vitro via a gastrointestinal tract simulation.

\section{Anti-obesity activities}

Animal studies have been conducted to assess the effects of chia seed and its oil on obesity. In obese rats, treatment with chia seed and chia seed oil resulted in similar effects with slight nuances. Poudyal et al. [20] summarized that insulin and glucose tolerance, visceral adiposity, hepatic steatosis, cardiac and hepatic fibrosis, and inflammation improved with chia seed supplementation. Chia seed inhibits SCD activity, which converts elaidic acid to conjugated linolenic acid. This study suggests that $C 18: 2 n-6$ is oxidized and transported into the mitochondria, which downregulates n-6/ $\mathrm{n}-3$ levels. The inhibition of SCD provides protection from murine obesity, cellular lipid accumulation, and insulin resistance [20]. As a background, n-3 fatty acid from fish oil decreases the effect of SCD-1 promoter. SCD-1 produces plasma palmitoleate, which in humans has been linked to hypertriglyceridemia and abdominal adiposity [20]. However, in this study, body weight gain, abdominal fat accumulation, and food intake were not reduced. Only the 6-wk treatment saw a reduction in HSP 60 and HSP70 levels, and changes were never seen with HSP25 and HSP90 levels. Glutathione peroxidase levels were only changed with chia oil treatment and never with chia seed treatment, and catalase, lipase, and DHFR levels were not altered by the treatment. Superoxide dismutase levels were increased but not to normal control levels. However, similar positive results were achieved in a study by Marineli et al. [21], which appears to support the proposed pharmacological pathway outlined by Poudyal et al. [20]. Marineli et al. [21] stated that, compared with the high-fructose group, chia did not reduce fat accumulation or weight gain but aided in glucose and insulin tolerance in obese rats. Their experiment was designed to allow the standard AIN-93 diet with protein concentra- 
tion at $12 \%$, which was given to the control group. The high-fructose group was administered $4 \%$ soybean oil, $31 \%$ lard, and $20 \%$ fructose. Chia seed groups received a high-fructose diet with $13.3 \%$ of chia seed, and the chia oil groups received $4 \%$ of this chia oil, which can be examined more closely within the context of blood lipid presence and hepatic enzyme activity.

\section{Activities on blood lipids and hepatic enzymes}

The administration of chia seed to rats resulted in the normalization to base levels of plasma AST and ALT. The data from Marineli's study [21] shows that the high-fat and high-fructose diet promoted increase in AST and ALT levels compared with the control. All groups treated with chia, except for the 6-wk-long chia seed treatment, showed a reduction in the concentration of AST and ALT (markers of liver damage) to base levels. Chia seed and oil did not reduce body weight gain or abdominal fat accumulation, but both treatments of chia improved glucose and insulin tolerance back to levels observed in lean animals. Chia oil in both treatments induced expression of skeletal muscle HSP 70 and HSP 25, while short treatment with chia seed increased expression only of skeletal muscle HSP 70. Chia oil in both treatments restored superoxide dismutase and glutathione peroxidase expression, previously made irregular by obesity. Long-term treatment with chia seed and short-term treatment with chia oil restored peroxisome proliferator-activated receptor coactivator-1a expression. Interestingly, this finding demonstrated that chia seed oil is better than chia seed at restoring the antioxidant system and oxidative energy metabolism and reducing liver damage in diet-induced obese rats. Chia seed and chia seed oil returned superoxide dismutase levels to normal, albeit lower than the control group [21]. Concurrent with the work submitted by Marineli, Ferreira et al. [22] were able to demonstrate that insulin levels and insulin sensitivity were improved by a diet of chia seed. Additionally, a significant decrease in visceral fat was expressed. Levels of thiobarbituric acid reactive substances and levels of carbonyl groups also decreased, and normalized levels of reactive oxygen species content and xanthine oxidase activity were observed. Basic leucine zipper gene NRF2 expression increased significantly compared with the control group [22]. However, the work by Marineli et al. did not show reduced body weight gain, levels of HSP90 were never changed, catalase and DHFR levels were not changed, and HSP25 was not modified in the 12-wk group, and HSP60 was unaffected in the short-term 6-wk study. Another rat-based study suggests that chia seeds may play a role in lipid redistribution by converting visceral fat, which has far more deleterious effects, into subcutaneous fat. Oleic acid levels were decreased with chronic alimentation of chia, which suggests that chia could possibly inhibit lipogenesis. In the same study, ingestion of chia seed restored to normal levels chronic low-grade inflammation in animals with metabolic syndrome, complimenting a study suggesting that the presence of (n-3) fatty acids and antioxidants in chia could inhibit an inflammatory response $[23,24]$. A study performed by Ayerza et al. [24] reports that the serum lipid profile in rats improved as rats displayed higher $n-3$ PUFA (18: 3n-3, 20 : 5n-3, and 22: 6n-3) plasma contents, and lower n-6 PUFA (18: 2n6 and 20: $4 n-6)$ contents, which can be reasonably predicted considering chia's high n-3/n-6 ratio. In these rats, high-density (or "good") cholesterol increased while triglyceride content decreased; however, the ratio between low-density lipoprotein to high-density lipoprotein content was not lowered. These findings bear weight in supporting the notion that chia supplementation improves cardiovascular health; however, like most reports, no change in body weight was recorded, nor were feed intake, feed conversion, liver weight, relative liver weight, hematocrit, hemoglobin, MCV, MCH, and MCHC values for the rats fed chia significantly changed. Da Silva et al. [25] also reported lower levels of low-density and very low-density cholesterol, as well as a reduction in triglyceride. Chia supplementation also improved hepatic factors by reducing liver weight and fat percentage; however, research by Chicco et al. [26] showed no significant difference in liver fat percentage.

In agreement with the work done by Ayerza et al. [24], Valenzuela et al. [27] found that ingesting vegetable oils containing high amounts of ALA caused a more dramatic accumulation of $n$ 3 long-chain PUFAs in Wistar rat plasma, liver, and adipose tissue, while DHA, total saturated fatty acid, and MUFA content was unchanged. However, the work of Valenzuela et al. [28] in 2014 showed a decreased n-6/n-3 ratio while EPA and DHA increased with the help of sacha inchi oil (Plukenetia volubilis L. [Euphorbiaceae]) and chia seed oil. Based on parameters tested in serum lipid profiles, chia was found to inhibit by either preventing or limiting dyslipidemia, liver triacyclglycerols that generate very lowdensity lipoprotein cholesterol, Fas ligands (which induce apoptosis and play a major role in pathogenesis of gastrointestinal diseases), acetyl coenzyme-A, glucose-6-phosphate dehydrogenase, and increased bioactivity involving fatty acid oxidation and carnitine palmitoyltransferase I. DHA was not modified in all tissues, total saturated fatty acid content was unaffected in the small intestine, and none of the values were modified in the brain [28]. Valenzuela et al. [29] again in 2016 reported an increased activity of carnitine palmitoyltransferase I in a study with rats, as well as an increase in the expression of the DNA transcription factor peroxisome proliferator-activated receptor- $\alpha$. The antioxidant status was increased as the chia-fed rats showed increased GSH/CSSG ratio and heightened superoxide dismutase activity, carnitine acylcarnitine transferase, and glutathione peroxidase. The inhibition of lipogenic enzymes fatty acid synthase and acetyl-CoA carboxylase was also demonstrated. However, plasma parameters of oxidative stress were also not modified by dietary ALA. The activity of these antioxidative enzymes was not modified in chia seed oil compared to the safflower group (initial and final body weight or hepatic and epididymal adipose tissue weight). No significant differences were observed for initial and final body weight or hepatic and epididymal adipose tissue weight during the experimental period of ALA intervention, and like most studies, body weight and weight of specific organs were unchanged. In the previously mentioned work by Valenzuela et al. [28] in 2014 regarding diverse tissue accretion of erythrocyte ALA, the authors concluded that DHA levels were not significantly modified in the kidney but referenced a Domenichiello et al. [30] study that claimed that rats fed ALA showed 100-times higher levels of DHA than the control. 


\section{In vivo effect of skeletal muscle}

After feeding chia to rats for three weeks, Chicco et al. [26] found no differences in the muscle triacyclglycerol content of rat gastrocnemius, but after 2 mo, the group treated with chia seed bore similarities to the control group in that parameter. In the same study, chia prevented alterations between the chia-treated and the control diet in the way of plasma triacyclglycerol and NEFA levels, which rose significantly with the control group alone.

\section{In vivo effect on body weight}

Valenzuela et al. [28] found that ingesting vegetable oils containing high amounts of ALA caused a more dramatic accumulation of $\mathrm{n}$-3 long-chain PUFA in Wistar rat plasma, liver, and adipose tissue. A high-fructose diet adjusted in protein and dietary fiber to match that of the chia seed was used in this case. The ratio of $n-6 / n-3$ decreased while EPA and DHA increased with the help of sacha inchi oil and chia oil (30.2\% makeup of seed), which included concentrations of ALA at $48 \%$ and $64 \%$, respectively. Rodents ingested less food while gaining more metabolic energy with the high-fructose diet than with the American Institute of Nutrition (AIN-93) diet. There were no significant differences in chia seed and chia seed oil diets in comparison with the high-fructose group in terms of superoxide dismutase activity. Concurrently, a study from Marineli et al. [31] had chia treatment groups showing reduced plasma thiobarbituric acid reactive substances and increased plasma glutathione levels in comparison to the high-fructose group. In comparison with AIN-93, chia treatment raised levels of liver glutathione peroxidase and glutathione reductase activity, as did the rats fed a high-fructose diet. In terms of liver and plasma antioxidant value, chia groups raised these levels in comparison with the standard diet, a reverse effect of high fructose. High-fructose diets encourage lipid peroxidation and weakened antioxidant capacity and enzyme activities involved in the antioxidant defense system in plasma and liver of rats. Catalase and glutathione reductase activity coupled with glutathione reduction occurred in conjunction with a chia diet, but superoxide dismutase and glutathione reductase activities were unaffected. A marker of oxidative stress, 8-isoprostane, is formed via free radical catalyzed peroxidation of arachidonic acid and indicates more specifically than any other reaction oxidative stress in vivo (see [21]). Dietary intake of chia prevents plasma 8-isoprostane increase in rats coupled with a high-fructose diet. All parameters of energy intake, visceral adiposity index, plasma metabolite levels, and glucose infusion rate returned to control values in the rats fed a sucrose-rich diet and when chia seed replaced chia oil for the last 3 mo of the feeding period. Body weight, superoxide dismutase levels, liver catalase, glutathione reductase, glutathione peroxidase, and thiobarbituric acid reactive substances activity did not return to normal levels along with the other conditions [31]. However, the aforementioned study by Chicco et al. [26] revealed a more normalized body weight against a sucrose-rich diet (energy intake was unchanged between the groups). Fortino et al. [32] also explored hepatic implications of chia in Wistar rats and concluded that chia improved the 18:0/16:0 fatty acid ratio and decreased SCD-1 index (which is directly correlated with triglyceride content). Most importantly, chia supplementation decreased
- Table 1 Chia seed and oil health benefits presented based on organ system and the corresponding biomarker in humans.

\begin{tabular}{|c|c|c|}
\hline Organ system & Biomarker & References \\
\hline \multirow[t]{11}{*}{ Cardiovascular } & Decreased blood glucose & {$[35-37]$} \\
\hline & Increased satiety & [38] \\
\hline & Increased plasma ALA & {$[38,41,48,49]$} \\
\hline & $\begin{array}{l}\text { Increased n-3/n-6 omega fatty } \\
\text { acid ratio }\end{array}$ & {$[38,41,48,49]$} \\
\hline & Increased plasma EPA & [38] \\
\hline & Increased plasma DPA & [38] \\
\hline & Increased plasma DHA & {$[38]$} \\
\hline & $\begin{array}{l}\text { Decreased body weight in } A B C A 1 \\
\text { R230C variant (MetS patients) }\end{array}$ & [39] \\
\hline & $\begin{array}{l}\text { Decreased adiponectin in } A B C A 1 \\
\text { R230C variant (MetS patients) }\end{array}$ & [39] \\
\hline & $\begin{array}{l}\text { Decreased weight in overweight } \\
\text { adults }\end{array}$ & {$[42]$} \\
\hline & $\begin{array}{l}\text { Decreased waist circumference } \\
\text { in overweight adults }\end{array}$ & {$[42]$} \\
\hline \multirow[t]{5}{*}{ Integumentary } & $\begin{array}{l}\text { Inhibition of total melanin } \\
\text { production }\end{array}$ & [43] \\
\hline & $\begin{array}{l}\text { Subjective improvement of } \\
\text { pruritic skin }\end{array}$ & [45] \\
\hline & $\begin{array}{l}\text { Improvement of lichen simplex } \\
\text { chronicus }\end{array}$ & [45] \\
\hline & Improvement of prurigo nodularis & [45] \\
\hline & Improvement of skin capacitance & [45] \\
\hline $\begin{array}{l}\text { Musculo- } \\
\text { skeletal }\end{array}$ & $\begin{array}{l}\text { Increased endurance in } \\
10 \mathrm{~K} \text { athletes }\end{array}$ & [47] \\
\hline
\end{tabular}

n-6/n-3 hepatic ratios but failed to significantly reduce visceral adiposity or incremental glucose levels within a 60 -min period.

\section{Human studies}

Antioxidant potential and obesity

With the awareness of Salvia's antioxidant potential, obesity and related issues associated with chia were readily analyzed in humans (see - Table 1), but glaring limitations remain. The review paper by de Souza et al. [33] concludes that the current state of research yields an inconclusive perspective on the potential of chia. However, tangible results have precipitated through multiple studies, and different research teams have successfully replicated results on occasion. Creus et al. [34] explored the normalizing potential of chia versus a sucrose-rich diet across such parameters as systolic blood pressure, body weight, and visceral adiposity, but no major differences in plasma metabolites, glucose infusion rate, and insulin levels for dyslipidemic rats were noted. Downregulation of intramyocardial lipid accumulation occurred with a combination of chia with sucrose-rich diet in these animals as well. Chia was also able to increase (to control levels) the percentage of active pyruvate dehydrogenase to $45.1 \%$ (compared to the sucrose group at $26.6 \%$ ), implying a more responsive metabolism, although the weight of the heart remained unaffected. 


\section{Postprandial glucose levels}

Additionally, the propensity toward overeating may be regulated with supplementation of chia. Lee et al. [35] found in one experiment that $S$. hispanica reduced the amount of postprandial blood glucose incremental area under the curve and reduced appetite on a subjective self-analysis by the subject. Vuksan et al. [36] proposed a similar analysis and demonstrated that ground chia can reduce the effect of postprandial glycemia, but they presented the limitation that this event may not occur with whole chia seed. This complements a 2010 study showing that chia seed supplementation lowered blood glucose levels but had no significant effect on subjective appetite $[35,36]$. Vuksan et al. [37] further extended knowledge in a 2016 study by demonstrating that postprandial glucose levels were reduced in flaxseed and chia seed supplements against the control, but there were no significant differences between the two seed groups for this category after $120 \mathrm{~min}$, although significant differences in values did appear after 30 min (with chia seed reducing glucose more effectively than flaxseed). The area under the curve values for glucose remained the same for both treatment groups.

\section{Cholesterol and body weight}

Significant weight loss occurred in postmenopausal women after 12 wk of milled chia seed intake, and serum concentration improved for total cholesterol, very low-density lipoprotein cholesterol, and high-density lipoprotein cholesterol, in agreement with the study of rat plasma by Ayerza and Coates [24]. Alternatively, Guevara-Cruz et al. [38] studied patients with metabolic syndrome and showed no significant trial differences in high-density lipoprotein or low-density lipoprotein cholesterol but did see a reduction in serum triglyceride after 2 mo of treatment. The specific genetic makeup may paint a picture of the propensity toward weight loss on the subjects, as those who expressed the $A B C A 1$ R230C gene experienced a more significant weight loss in the chia group than those who expressed the $A B C A 1 \mathrm{R} 230 \mathrm{C}$ without chia supplementation. Dietary intake of ALA, EPA, DPA, and DHA fatty acids, as well as subjective overall health perception, were unchanged during the 7-wk study [38]. Waist circumference in the Ayerza and Coates study [39] was reduced as well, but the limitation exists that all three of these categories of values only improved in subjects whose lipid profiles were previously abnormal. Further supporting the trend of only ameliorating abnormal conditions is another study by this same group in pigs, which concluded that, for these animals, waist circumference and body weight were unaffected by chia alimentation, which may reveal that certain aspects of chia may only be effective for murine alimentation [38].

\section{Diabetes}

Vuksan et al. [40] extended research to type 2 diabetes and in 2016 concluded that chia increased the process of weight loss by $1.6 \mathrm{~kg}$ and waist circumference by $2.4 \mathrm{~kg}$ in type 2 diabetic patients against the control. The limitation exists that the phenomenon of chia coming to lessen the degree of abnormal indices of body weight and fat accumulation while not improving values already in normal ranges is an occurrence manifest in both human and animal studies. Subjective health characteristics, serum C-reactive protein and cytokine levels, were unchanged as well.

\section{Criticism on reputed human studies}

Contradicting conclusions confuse the legitimacy of the reports addressing chia's potential to alter risk factors for cardiovascular disease. One review paper from 2015 summarizes that the effects of chia seed consumption are inconclusive, reporting that bias, inadequate sample sizes, and faulty experimental design between the work of Vuksan et al. [36], Nieman et al. [41], Lee et al. [35], and jin et al. [42]. The work by de Souza et al. [32] highlighted risk of implicit bias, faulty experimental design, and inadequate sample size. Guevara-Cruz et al. [38], Ho et al. [35], Nieman et al. [42], jin et al. [42], Vuksan et al. [36], and Nieman et al. [41] were assessed in the review by de Souza et al. [32] and were found to contain technical errors. Since the most recent work mentioned above Lee et al. [35], five reports on cardiovascular risk factors have been included in the review herein. The following aims to assess the legitimacy of those reports called into question by de Souza et al. [32], on the basis of experimental replicability. For example, de Souza et al. [32] questioned the legitimacy of the conclusion that ALA levels in reports by Nieman et al. [41] and jin et al. [42] and postprandial glucose levels in reports by Vuksan et al. [36] and Lee et al. [35] were improved. However, Vuksan et al. [37] completed further studies to conclude that postprandial glucose levels were improved and waist circumference decreased in separate studies in 2017. The work done by de Souza et al. [32] is a necessary effort to ascertain legitimacy of claims of chia's aid toward human health, especially cardiovascular risk factors. More such studies are needed to separate fact from conjecture when it comes to chia's potential to improve human health.

\section{End-Stage Renal Disease}

\section{Human studies}

The research behind possible benefits of chia seed intake extends to topical administration of chia compounds on cutaneous systems as they relate to end-stage renal disease (see $>$ Table 1 ). Chia seed extract is demonstrated to act as an inhibitor of melanin biosynthesis in Melan-a positive dermal cells, and when combined with pomegranate fruit extract, it had a synergistic effect on inhibition with no effect on tyrosinase activity. Chia downregulated expression of melanogenesis-related genes (Tyr, Tyrp1, and Mc1r) on its own and when combined with pomegranate fruit extract. Diwarkar et al. [43] suggested that tyrosinase activity was not responsible for this inhibition, and linolenic and ALA did not significantly impact melanin production in Melan-a cells. A patent application of $1-10 \%$ of pure chia seed oil reports a moisturizing effect, while also treating atopic dermatitis, contact dermatitis, ichthyosis, chronic eczema, psoriasis, and xerosis accompanied by pruritus caused by chronic renal failure and diabetes mellitus. Similarly, after 8 wk of application of chia seed oil, significant improvements in skin hydration, lichen simplex chronicus, and prurigo nodularis were observed in all patients [44]. A similar improvement was also observed among healthy volunteers with xerotic pruritus in the same study. Improvement of epidermal permeabil- 
ity barrier function and skin hydration, represented by transepidermal water loss and skin capacitance, respectively, were also observed. No adverse effects were observed in all the tested patients and volunteers [44]. The conclusion drawn was that chia seed oil can be used as an adjuvant moisturizing agent for pruritic skin, including that of end-stage renal disease patients, but in a study by Jeong et al. [45], transepidermal water loss values were unchanged, as were skin capacitance and skin surface $\mathrm{pH}$.

\section{Athletic Performance}

\section{Human studies}

Nieman et al. [46] performed a study of athletes that showed increased levels of plasma ALA with chia seed oil $(7 \mathrm{kcal} / \mathrm{kg}$ ) use compared to water alone. The athletes also expressed major increases in total leukocyte counts, plasma cortisol, and plasma cytokines with no trial difference [46]. The results of this study found near doubled plasma ALA levels (82\% increase with seed, $91 \%$ increase with oil) within $2.5 \mathrm{~h}$. These levels stayed elevated for several hours. Levels of ALA then fell to pre-ingestion levels without conversion to EPA or DHA within $24 \mathrm{~h}$ compared to snack clusters not containing chia, resulting in an inconclusive picture of chia's effect on running performance. The primary argument by Nieman et al. [46] is that run time to exhaustion alter respiratory exchange ratio or counter elevations in cortisol and inflammatory outcome measures, which were not improved, although immediate post-run ALA levels did increase dramatically. Illian et al. [47] performed a strategic study in runners and concluded that chia oil glycogen loading could be used in place of omega-3 loading but ultimately produced no discernible benefits (see $>$ Table 1 ). The strategic benefit of using chia supplementation is that it allows for less sugar intake.

\section{Effects in Postmenopausal Women}

Increased levels of ALA have been associated with multiple health benefits. A supplement of $25 \mathrm{~g}$ of daily milled chia seed supplementation over a 7-wk period increased plasma ALA levels by $138 \%$ when compared to baseline. Plasma EPA rose $30 \%$ above baseline. An insignificant decrease in DHA levels occurred over the 7-wk study. Subjects in this study were postmenopausal women between 17 and $29 \mathrm{~kg} / \mathrm{m}^{3}$ [48]. In overweight women, $25 \mathrm{~g} / \mathrm{d}$ supplementation of milled and whole chia seed over $10 \mathrm{wk}$ was found to improve levels of ALA and EPA (an omega-3 fatty acid) against the placebo group, which ingested poppy seed, but did not improve body composition, inflammation, blood pressure, augmentation index, and lipoprotein levels [49].

\section{Adverse Events}

Current research on chia produces a rather incomplete picture on the risk profile of its ingestion. Chia extract was prepared from the seeds of a commercial product and analyzed by SDS-PAGE, immunoblot, and identification of proteins by MS/MS to determine potential allergens. Chia allergens involved are water-soluble and lipid-soluble and include a lectin, an elongation factor, and an $11 \mathrm{~S}$ globulin as known allergens in addition to another three as yet undescribed allergens. The negative IgE determinations to legumins, vicillins, and conglutins included in the ISAC platform suggests that the chia allergens described have no cross-reactivity with these proteins. This information suggests that there is potential for an allergic reaction from chia seed and its oil through an $\mathrm{gE}$ reaction [50]. Jimenez et al. [50] also reports a case of a 54-yearold male subject developing an allergic reaction from intake of chia seeds, who also tested positive for skin prick tests for pollen, profilin, and cat dandruff. The Scientific Panel of Dietetic Products of the European Food Safety Authority relates sesame allergy to chia allergy and that the proteins were sensitive to proteolysis [51]. It was also emphasized that cross-reactivity cannot be ruled out [51]. The application for chia status with regard to availability and safety by Robert Craig \& Sons Ltd. found no relevant link between chia seed ingestion and allergic activity, even at levels far exceeding convention [52]. Similarly, the application by Jean-Louis Bresson [53] to the European Food Safety Authority concluded that the proposed usage of chia seeds should not result in adverse effects.

\section{Conclusion}

The effects of chia on cardiovascular health have seen extensive increases of knowledge through a number of research studies. However, most of this research has been carried out with animals, and there are still limitations that exist in understanding the effect of chia supplementation on human cardiovascular health. Research related to chia supplementation results on other health endpoints, athletic performance, apoptotic anticancer activity, women's health, pruritic skin relief, and others is either too limited or preliminary to make any substantive conclusions at this time.

\section{Acknowledgements}

The authors are indebted to Auburn University Initiative in Cancer for financial support. Also, the authors are grateful to Dr. Robin Marles from Health Canada for providing helpful critiques to the manuscript.

\section{Conflict of Interest}

The authors declare no conflict of interest.

\section{References}

[1] Ali NM, Yeap SK, Ho WY, Beh BK, Tan SW, Tan SG. The promising future of chia, Salvia hispanica L. J Biomed Biotechnol 2012; 2012: 171956

[2] United States Pharmacopeal Convention 2017. USP40-NF35 Supplement: No. 2 Page 8889. Available at http://www.uspnf.com/official-text. Accessed December 1, 2017

[3] Kintzios SE. Sage: The Genus Salvia. Boca Raton, FL: CRC Press; 2000: 9

[4] Health Canada. Salvia hispanica. Available at http://webprod.hc-sc.gc.ca/ nhpid-bdipsn/ingredReq.do?id=6672\&lang=eng. Accessed September 18, 2017

[5] Application for the authorization of chia seed from Salvia hispanica L. for consumption as a food and as an ingredient in additional food groups. Available at https://acnfp.food.gov.uk/sites/default/files/mnt/drupal_ data/sources/files/multimedia/pdfs/applicdosschiacompany.pdf. Accessed November 5, 2016 
[6] Uribe JAR, Perez JIN, Kauil HC, Rubio GR, Alcocer CG. Extraction of oil from chia seeds with supercritical CO2. J Supercrit Fluids 2011; 56: 174-178

[7] Ullah R, Nadeem M, Khalique A, Imran M, Mehmood S, Javid A, Hussain J. Nutritional and therapeutic perspectives of Chia (Salvia hispanica L.): a review. J Food Sci Technol 2016; 53: 1750-1758

[8] US Department of Agriculture. USDA National Nutrient Database for Standard Reference. Available at https://ndb.nal.usda.gov/ndb/. Accessed September 18, 2017

[9] Imran M, Nadeem M, Manzoor MF, Javed A, Ali Z, Akhtar MN, Ali M, Hussain Y. Fatty acids characterization, oxidative perspectives and consumer acceptability of oil extracted from pre-treated chia (Salvia hispanica L.) seeds. Lipids Health Dis 2016; 15: 162

[10] Connor WE. Importance of n-3 fatty acids in health and disease. Am J Clin Nutr 2000; 71: 171-175

[11] da Silva BP, Anunciaciao PC, da Silva Matyelka J, Della Lucia CM, Martino HSD, Pinheiro-Sant'Ana HM. Chemical composition of Brazilian chia seeds grown in different places. Food Chem 2016; 221: 1709-1716

[12] Espada CE, Berra MA, Martinez MJ, Eynard AR, Pasqualini ME. Effect of Chia oil (Salvia hispanica) rich in $\omega$-3 fatty acids on the eicosanoid release, apoptosis and T-lymphocyte tumor infiltration in a murine mammary gland adenocarcinoma. Prostaglandins Leukot Essent Fatty Acids 2007; 77: $21-28$

[13] Rosas-Ramirez DG, Fragoso-Serrano M, Escandon-Rivera S, VargasRamirez AL, Reyes-Grajeda JP, Soriano-Garcia M. Resistance-modifying activity in vinblastine-resistant human breast cancer cells by oligosaccharides obtained from mucilage of chia seeds. Phytother Res 2017; 31: 906-914

[14] Fernandez I, Vidueiros SM, Ayerza R, Coates W, Pallaro A. Impact of chia (Salvia hispanica L.) on the immune system: preliminary study. Proc Nutr Soc 2008; 67: E12

[15] Rahman U, Nadeem M, Imran M. Omega-3 fatty acids and oxidative stability of ice cream supplemented with olein fraction of chia (Salvia hispanica L.) oil. Lipids Health Dis 2017; 16: 34

[16] Segura-Campos MR, Salazar-Vega IM, Chel-Guerrero LA, BetancurAncona DA. Biological potential of chia (Salvia hispanica L.) protein hydrolysates and their incorporation into functional foods. Food Sci Technol 2013; 50: 723-731

[17] Segura-Campos MR, Gonzalez FP, Guerrero LC, Ancona DB. Angiotensin I-converting enzyme inhibitory peptides of chia (Salvia hispanica) produced by enzymatic hydrolysis. Int J Food Sci 2013; 2013: 158482

[18] Salazar-Vega IM, Segura-Campos MR, Chel-Guerrero LA, BetancurAncona DA. Antihypertensive and antioxidant Effects of functional Foods containing Chia (Salvia hispanica) Protein Hydrolysates. In: Valdez B, ed. Scientific, Health and social Aspects of the Food Industry. Rijeka, Croatia: InTech; 2012: 381-398

[19] Orona-Tamayo D, Valverde ME, Nieto-Rendon B, Paredes-Lopez O. Inhibitory activity of chia (Salvia hispanica L.) protein fractions against angiotensin I-converting enzyme and antioxidant capacity. Food Sci Technol 2015; 64: 236-242

[20] Poudyal H, Panchal SK, Waanders ], Ward L, Brown L. Lipid redistribution by $\alpha$-linolenic acid-rich chia seed inhibits and induces cardiac and hepatic protection in diet-induced obese rats. J Nutr Biochem 2012; 24: 153162

[21] Marineli R, Moura C, Moraes E, Lenquiste S, Lollo PC, Morato P, AmayaFarfan J, Marostica MR jr. Chia (Salvia hispanica L.) enhances HSP, PGC- $1 \alpha$ expressions and improves glucose tolerance in diet-induced obese rats. Nutrition 2015; 51: 740-748

[22] Ferreira MR, Alvarez SM, Illesca M, Giménez MS, Lombardo YB. Dietary Salba (Salvia hispanica L.) ameliorates the adipose tissue dysfunction of dyslipemic insulin-resistant rats through mechanisms involving oxidative stress, inflammatory cytokines and peroxisome proliferator-activated receptor $\gamma$. Eur J Nutr 2018; 57: 83-94
[23] Flachs P, Rossmeisl M, Bryhn M, Kopecky ]. Cellular and molecular effects of $n-3$ polyunsaturated fatty acids on adipose tissue biology and metabolism. Clin Sci (Lond) 2009; 116: 1-16

[24] Ayerza R jr., Coates W. Effect of dietary $\alpha$-linolenic fatty acid derived from chia when fed as ground seed, whole seed and oil on lipid content and fatty acid composition of rat plasma. Ann Nutr Metab 2007; 51: $27-$ 34

[25] Da Silva BP, Dias DM, de Castro Moreira ME, Toledo RCL, da Matta SLP, Lucia CMD, Martino HSD, Pinheiro-Sant'Ana HM. Chia seed shows good protein quality, hypoglycemic effect and improves the lipid profile and liver and intestinal morphology of wistar rats. Plant Foods Hum Nutr 2016; 71: 225-230

[26] Chicco AG, D’Alessandro ME, Hein G], Oliva ME, Lombardo YB. Dietary chia seed (Salvia hispanica L.) rich in alpha-linolenic acid improves adiposity and normalises hypertriacylglycerolaemia and insulin resistance in dyslipaemic rats. Br J Nutr 2008; 101: 41-50

[27] Valenzuela R, Barrera C, Ayala JM, Sanhueza J, Valenzuela A. Vegetable oils rich in alpha linolenic acid allow a higher accretion of n-3 LCPUFA in the plasma, liver and adipose tissue of the rat. Grasas Aceites 2014; 65: 26-28

[28] Valenzuela RB, Barrera CR, Gonzalez-Astorgo M, Sanhueza JC, Valenzuela $A B$. Alpha linolenic acid (ALA) from Rosa canina, sacha inchi and chia oils may increase ALA accretion and its conversion into n-3 LCPUFA in diverse tissues of the rat. Food Funct 2014; 5: 1564

[29] Valenzuela R, Rincón-Cervera MÁ, Hernandez-Rodas MC, Barrera C, Espinosa A, Marambio M, Valenzuela A. Vegetable oils rich in alpha linolenic acid increment hepatic n-3 LCPUFA, modulating the fatty acid metabolism and antioxidant response in rats. Prostaglandins Leukot Essent Fatty Acids 2016; 111: 25-35

[30] Domenichiello AF, Chen CT, Trepanier MO, Stavro PM, Bazinet RP. Whole body synthesis rates of DHA from $\alpha$-linolenic acid are greater than brain DHA accretion and uptake rates in adult rats. J Lipid Res 2014; 55: 62-74

[31] Marineli R, Lenquiste SA, Moraes EA, Marostica MR jr. M. Antioxidant potential of dietary chia seed and oil in diet-induced obese rats. Food Res Int 2015; 76: 666-674

[32] Fortino MA, Oliva ME, Rodriguez S, Lombardo YB, Chicco A. Could postweaning dietary chia seed mitigate the development of dyslipidemia, liver steatosis and altered glucose homeostasis in offspring exposed to a sucrose-rich diet from utero to adulthood? Prostaglandins Leukot Essent Fatty Acids 2017; 116: 19-26

[33] de Souza Ferreira C, de Sousa Fomes LdeF, da Silva GES, Rosa G. Effect of chia seed (Salvia hispanica L.) consumption on cardiovascular risk factors in humans: a systematic review. Nutr Hosp 2015; 32: 1909-1918

[34] Creus A, Ferreira MR, Oliva ME, Lombardo YB. Mechanisms involved in the improvement of lipotoxicity and impaired lipid metabolism by dietary $\alpha$-linolenic acid rich Salvia hispanica L. (Salba) seed in the heart of dyslipemic insulin-resistant rats. J Clin Med 2016; 5: E18

[35] Lee AS, Jovanovski E, Jenkins AL, Desouza R, Vuksan V. Effect of whole and ground Salba Seeds (Salvia hispanica L.) on postprandial Glycemia in healthy Volunteers: a randomized controlled, dose-response Trial [Thesis]. Toronto, Canada: University of Toronto; 2009

[36] Vuksan V, Jenkins AL, Dias AG, Lee AS, Jovanovski E, Rogovik AL, Hanna A. Reduction in postprandial glucose excursion and prolongation of satiety: possible explanation of the long-term effects of whole grain Salba (Salvia hispanica L.). Eur J Clin Nutr 2010; 64: 436-438

[37] Vuksan V, Choleva L, Jovanovski E, Jenkins AL, Au-Yeung F, Dias AG, Ho HVT, Zurbau A, Duvnjak L. Comparison of flax (Linum usitatissimum) and Salba-chia (Salvia hispanica L.) seeds on postprandial glycemia and satiety in healthy individuals: a randomized, controlled, crossover study. Eur J Clin Nutr 2017; 71: 234-238

[38] Guevara-Cruz M, Tovar AR, Aguilar-Salinas CA, Medina-Vera I, GilZenteno L, Hernandez-Viveros I, Lopez-Romero P, Ordaz-Nava G, Canizales-Quinteros S, Pineda LEG, Torres N. A dietary pattern including nopal, chia seed, soy protein, and oat reduces serum triglycerides and 
glucose intolerance in patients with metabolic syndrome. J Nutr 2012; 142: 64-69

[39] Coates W, Ayerza R. Chia (Salvia hispanica L.) seed as an n-3 fatty acid source for finishing pigs: effects on fatty acid composition and fat stability of the meat and internal fat, growth performance, and meat sensory characteristics. J Anim Sci 2009; 87: 3798-3804

[40] Vuksan V, Jenkins AL, Brissette C, Choleva L, Jovanovski E, Gibbs AL, Bazinet RP, Au-Yeung F, Zurbau A, Ho HVT, Duvnjak L, Sievenpiper JL, Josse RG, Hanna A. Salba-chia (Salvia hispanica L.) in the treatment of overweight and obese patients with type 2 diabetes: a double-blind randomized controlled trial. Nutr Metab Cardiovasc Dis 2017; 27: 138-146

[41] Nieman DC, Cayea EJ, Austin MD, Henson DA, McAnulty SR, Jin F. Chia seed does not promote weight loss or alter disease risk factors in overweight adults. Nutr Res 2009; 26: 411-414

[42] Jin F, Nieman DC, Sha W, Xie G, Qiu Y, Jia W. Supplementation of milled chia seeds increases plasma ala and epa in postmenopausal women. Plant Foods Hum Nutr 2012; 76: 105-110

[43] Diwakar G, Rana J, Saito L, Vredeveld D, Zemaitis D, Scholten J. Inhibitory effect of a novel combination of Salvia hispanica (chia) seed and Punica granatum (pomegranate) fruit extracts on melanin production. Fitoterapia 2014; 97: 164-171

[44] Park BD, Jung SG, Park HJ. Skin external composition containing chia seed oil. Korea Patent 2010005799; 2010

[45] Jeong SK, Park HJ, Park BD, Kim IH. Effectiveness of topical chia seed oil on pruritus of end-stage renal disease (esrd) patients and healthy volunteers. Ann Dermatol 2010; 22: 143-148

[46] Nieman DC, Gillitt ND, Meaney MP, Dew DA. No positive influence of ingesting chia seed oil on human running performance. Nutrients 2015; 7: 3666-3676
[47] Illian TG, Casey JC, Bishop PA. Omega 3 chia seed loading as a means of carbohydrate loading. J Strength Cond Res 2011; 25: 61-65

[48] Valenzuela R, Buscanan KA, Chamorro R, Barrera C, Sandoval J, Puigrredon C, Parraguez G, Orellana P, Gonzalez V, Valenzuela A. Modification of docosahexaenoic acid composition of milk from nursing women who received alpha linolenic acid from chia oil during gestation and nursing. Nutrients 2015; 7: 6405-6424

[49] Nieman DC, Gillitt N, Jin F, Henson DA, Kennerly K, Shanely RA, Ore B, Su $\mathrm{M}$, Schwartz $\mathrm{S}$. Chia seed supplementation and disease risk factors in overweight women: a metabolomics investigation. J Altern Complement Med 2012; 18: 700-708

[50] Garcia Jimenez S, Pastor Vargas C, de las Heras M, Sanz Maroto A Vivanco F, Sastre J Allergen characterization of chia seeds (Salvia hispanica), a new allergenic food. J Investig Allergol Clin Immunol 2015; 25: 55-56

[51] European Food and Safety Authority. Opinion of the scientific panel on dietetic products, nutrition and allergies on a request from the commission related to the safety of chia (Salvia hispanica L.) seed and ground whole chia seed as a novel food ingredient intended for use in bread. EFSA J 2005; 278: 1-12

[52] Armstrong D. Opinion on an application under the novel foods regulation for chia (Salvia hispanica L.). Advisory Committee for Novel Foods and Processes; 2004. Available at https://acnfp.food.gov.uk/sites/ default/files/mnt/drupal_data/sources/files/multimedia/pdfs/ chiaappinvagrop.pdf. Accessed March 7, 2018

[53] Bresson JL. Opinion on the safety of 'Chia seeds (Salvia hispanica L.) and ground whole Chia seeds' as a food ingredient. EFSA J 2009; 996: 1-26 